\title{
Using nanoHUB.org for Teaching and Learning Nanoelectronic Devices in Materials Engineering
}

\author{
A Simulation-based Educational Approach to the Study of Carbon Nanotubes
}

\author{
José M. de la Rosa \\ Institute of Microelectronics of Sevilla, IMSE-CNM (CSIC/Universidad de Sevilla) \\ C/ Américo Vespucio, Parque Tecnológico de la Cartuja, 41092 Sevilla, SPAIN \\ E-mail: jrosa@imse-cnm.csic.es
}

\begin{abstract}
This paper presents an educational methodology that uses the computational resources available in the nanoHUB.org online research and education platform. To this end, a number of software tools provided by nanoHUB.org have been incorporated as part of the practical lab exercises and linked to an e-learning virtual environment of a course on Electronic Materials, with emphasis on Nanoelectronic devices. The activities carried out during the course allowed students to enhance their understanding of those theoretical concepts dealing with Nanoelectronic materials and devices, thus becoming more motivated and satisfied. As an application and example, a set of experiences carried out by students is described. These experiences cover different Nanoelectronic materials and devices, going from physical and theoretical principles to device simulation. The proposed method has been applied to an undergraduate course, although it could be extended also to master students enrolled in courses dealing with materials science and engineering.
\end{abstract}

Keywords: Nanoelectronics; nanoHUB.org; carbon nanotubes.

\section{INTRODUCTION}

The use of computer and web-based educational and research software is becoming a common practice to improve the quality of teaching and learning in engineering courses. Within a period of just a few years, teaching methodology has changed radically in Science and Engineering courses, where classical blackboard-based explanations are being complemented and sometimes replaced altogether by new teaching techniques. These new procedures range from simply using a laptop or an iPad-style tablet to project transparencies onto the board to virtualizing subjects by means of e-learning platforms such as Web Course Tools (WebCT) [1].

One of the most successful examples of using e-learning methodologies is nanoHUB.org - a freely online platform for research and education, which was developed by the National Science Foundation (NSF) in the United States as an interuniversity network for computational nanotechnology [2]. Its numerous multimedia resources include learning modules, podcasts, class notes and transparencies, and it also has a wide range of online CAD simulation tools, which are executed on a distributed computing network and can be accessed via Internet without the need for any type of locally installed software [3].

The aforementioned computational resources provided by nanoHUB.org constitute a unique powerful tool for both researchers, educators and students in order to get a deep insight about the theoretical principles and applications of the new emerging materials and devices used in Nanotechnology. However, from a pedagogical perspective, and even though nanoHUB.org is very well organized and managed, it is important to guide students in order to help them browsing through the enormous amount of multimedia data they can access. This is particularly critical for those students pursuing engineering not so related to electronic devices, as for instance in the case of materials engineering undergraduate students.

In this scenario, this paper describes a systematic approach to use nanoHUB.org for studying Nanoelectronic devices in an undergraduate course named Electronic Materials (EM). Several extra teaching resources have been incorporated in the course in order to complement both lecture notes, slide presentations and lab exercises. These resources - extracted from the multimedia resources provided by nanoHUB.org - are properly organized by the topics covered in the course dealing with Nanotechnology, namely: carbon-based electronic devices, heterostructures, resonant tunneling devices, singleelectron devices, quantum and spintronic devices, etc. The underlying principles and practical issues behind all these topics are systematically studied by properly combining traditional teaching methods with the nanoHUB.org facilities. As an illustration, several students' experiences with nanoHUB.org are overviewed and the incorporation of the generated didactic materials into the course WebCT platform is described as part of the presented methodology.

\section{COURSE DESCRIPTION AND OBJECTIVES}

The EM course belongs to a bachelor degree program on Materials Engineering at the University of Seville. The course is given in a 15-week class period, and corresponds to six European Credit Transfer System (ECTS) - three of them being theoretical ECTS credits and the other three ones corresponding to practical ECTS credits. The contents of the course are organized around five units:

- Unit I: Introduction to electronics materials

- Unit II: Fundamentals of semiconductor materials

- Unit III: Micro/Nanotechnologies and Fabrication

- Unit IV: Overview of Microelectronic devices

- Unit V: Overview of Nanoelectronic devices

This work has been supported in part by the Spanish Ministry of Science and Innovation (with support from the European Regional Development Fund) under contracts TEC2007-67247-C02-01/MIC, TEC2010-14825/MIC, in part by the Consejería de Innovación, Ciencia y Empresa, under contract TIC-2532 and in part by the I Plan Propio de Docencia de la U. de Sevilla, LabCMA2010 project. 
Essentially, the main objective of the course is to give an overview of the materials used in Microelectronics and Nanoelectronics, putting emphasis on their physical properties, characterization and fabrication processes, as well as their application to Micro- and Nanoelectronic devices. Bering this general objective in mind, the activities described in this paper are structured around two fundamental objectives: firstly, the use of the virtual platform nanoHUB.org by EM students as a means of reinforcing and furthering their knowledge of the materials and devices employed in Nanoelectronics; and secondly, the incorporation of the newly acquired teaching and learning resources as part of the EM core content within the WebCT platform.

To make the most of the nanoHUB.org platform, this teaching experience proposed a number of practical exercises, which were then carried out by EM students both in a computer lab room available to them during class time and outside class time by accessing nanoHUB.org directly from their own personal computers. The activities proposed attempted to cover most of the subjects syllabus, and especially those areas related to the materials used in Nanoelectronics. Each piece of work produced by the students was to cover a different topic. These topics, as will be detailed later, included nanomanufacturing processes, heterostructure-based devices, quantum confinement, carbon nanotubes, spintronic materials and devices, etc. The idea was thus to encourage and increase the use of advanced CAD tools for analyzing, designing and characterizing the materials employed in Nanoelectronics through telematic access to the computing resources and virtual laboratories available at nanoHUB.org.

The overall objective described above can be divided into the following partial objectives:

- To encourage active student participation using the tools available in the WebCT virtual teaching platform. To this end an attempt was made to synchronize the activities to be carried out with the syllabus being followed in class throughout the academic year.

- Remote access to CAD and iLabs tools available in the nanoHUB.org virtual platform. An individual user account was created in the platform for each student, enabling them to use nanoHUB.org as a WebCT specific aid when studying Nanoelectronics-related materials.

- Creation and updating of didactic material on the WebCT platform, at different levels. Apart from the class notes and other teaching resources gradually being added by the teacher, this also included complementary material such as computer simulations, videos, technical documents, links to nanoHUB web pages, etc.

- Use of bibliographical databases related to the Materials Engineering degree course, above all those dealing with Electronics in general and Nanoelectronics in particular. Specifically, the databases used were those of IEEE Xplore and nanoHUB.org itself.

\section{PROPOSED Methodology}

The working methodology presented in this work is fueled by the following lines of teaching improvement:

- $\quad$ Experience with new teaching methodologies, because the proposal was to change traditional teaching methodology, based on laboratory procedures and subject to the usually limited resources available for the same - especially in cutting edge technologies such as Nanoelectronics, for an experience centred on the use of telematic computing and laboratory resources also known as $i$-Labs. The virtual platform chosen nanoHUB.org - constitutes a unique environment for the methodology developed in this work.

- Methodological change geared towards the acquisition of complementary skills. In this work, the teaching objective was to improve the learning experience of EM students through the development of transversal and complementary skills, such as analysis, synthesis, organization and planning, a solid professional grounding, the ability to analyze data from different sources, decision-taking, teamwork, interpersonal skills, the capacity to apply theory to practical situations, research skills, etc.

- Encouragement of the use of active teaching methodologies, because the proposal addresses the study of materials, nanodevices and Nanoelectronic manufacturing processes (one of the main course objectives in this subject) using the most advanced tools yet to have been developed in the world's leading universities and research institutions and which are now available for academic use on the nanoHUB.org platform.

- Design of interdisciplinary and hands-on teaching activities. In this particular case, $50 \%$ of the total course content is practical. This teaching improvement activity therefore aims at helping students not only to put their theoretical knowledge into practice but also to develop complementary skills, which will enable them to perform their professional tasks correctly.

All these innovative teaching approaches were integrated into the methodology and the evaluation system of the subject being studied. To provide a focal point for our study and to employ the nanoHUB.org platform in the most efficient, beneficial manner possible, a series of topics was established as a basis for the proposed activities to be carried out by the students. These work proposals were arranged as follows.

Projects were proposed to be carried out either individually or in groups. Each proposal focused on one of the topics covered in the subject's teaching units, with particular emphasis being placed on content related to Nanoelectronics. For each topic, the resources available in nanoHUB.org were analyzed, namely: tutorials, courses, simulation tools, i-lab instrumentation, links, bibliographical sources and resources, etc. A report was written by students for each project carried out, including an introduction, the content, a description of the 
computing resources employed and the results obtained using them.

Class time devoted to practical class sessions ( 2 hours a week) was concentrated at the end of the four-month teaching period and was mainly used to check the work being done by the students with the nanoHUB.org platform. The students explained their progress so far and described the different problems they were finding. For most students, the marks given for the project reports they handed resulted in improving their final grade, thanks principally to the quality of the reports and their relevance to the proposed objectives.

\section{RESULTS}

The results of this teaching improvement work can be divided into two groups or categories:

- Results obtained by students as part of their experience using the nanoHUB.org platform.

- New teaching material added to the WebCT, related to the contents and resources of the nanoHUB platform.

The main results obtained in each of these activities are described below.

\section{A. Students' experience with nanoHUB.org}

The work done by the students on the nanoHUB.org platform was structured around the following topics:

- Nanomanufacturing processes and techniques

- Nanoelectronic devices based on quantum confinement

- Resonant tunelling devices

- $\quad$ Spintronic materials and devices

- Carbon nanotubes

For each topic, students reported the resources available in nanoHUB.org, such as presentations, course notes, CAD simulation tools, animations, learning modules, bibliographical references, links to pages, etc. As an example, some of the results reported in some of the projects are summarized below.

1) New nanomanufacturing processes and techniques: This work focussed on searching for resources regarding new techniques for manufacturing Nanoelectronic materials and devices. One of the resources found by the students engaged on this project was a course featuring a conveniently classified description of new photolithographic techniques used in the manufacture of nanostructures and nanodevices [4]. Some of theses contents are being incorporated into the syllabus for the present academic year 2011-2012.

2) Nanoelectronic devices based on quantum confinement: Apart from explaining techniques for preparing and manufacturing Nanoelectronic devices based on quantum confinement, in this project students also analysed the multimedia resources available on the nanoHUB.org platform for simulating and studying those devices. One particularly interesting resource is the remote computing tool Quantum Dot Lab [5], with which it is possible to calculate and graphically represent the wave functions and energy levels of a quantum dot and numerically solve the Schrdinger equation for that dot. The application has a very user-friendly interface, making it especially suitable for teaching and learning purposes in the corresponding lesson of the course subject being studied in this work (EM).

3) Carbon NanoTubes (CNTS): This activity was aimed at studying available CAD tools for the analysis and simulation of CNTs. More specifically, the students involved analyzed the following tools at nanoHUB.org:

- Cristal Viewer, which is very useful for displaying an SWCNT type CNT in three dimensions [6].

- CNTbands, which produces diagrams of CNT energy bands in accordance with the principal structural characteristics of CNTs, for example, its diameter, length and chiral vector [7].

- $\quad$ CNT Mobility, which simulates carrier mobility in a CNTFET transistor [8].

- CNT FET Lab, a tool which can be used to obtain the characteristics of a FET transistor made with CNTs [9].

- $\quad$ FETToy, which simulates FET devices made using different materials and technologies, including CNTs [10].

For all of them, students reported a user guide, including some examples that illustrate how to use these tools to put in practice the theoretical contents studied in classroom.

\section{B. Incorporation of the generated didactic materials into WebCT platform}

The activities carried out as part of this educational research experience have contributed to a significant improvement in the materials and resources available on the WebCT for the course subject of study in this work. The virtualization of this course on WebCT - which began prior to the teaching activity presented in this work - includes presentations, notes, and practical exercises for self-correction by students. Thus, this work has focused on incorporating new contents relevant to those parts of the course dealing with Nanoelectronics. Specifically, a new learning module on carbon nanotubes has been added. The new module includes the following sections: structure and properties, synthesis, devices, manufacturing processes and CAD tools.

Fig. 1 shows some screenshots of the WebCT showing links to nanoHUB.org and the windows of applications and CAD tools that students registered for this course can access directly from the WebCT platform.

\section{STUDENTS' FEEDBACK}

Students attending the ME course were surveyed in order to give us their feedback to improve the quality of the course in terms of contents, learning resources and pedagogic procedures. Fig. 2(a) shows the interest and motivation of students for the course contents. More than $55 \%$ of them expressed a 
very good motivation level, while over $33 \%$ showed the highest level of motivation and interest. Students were also asked about their overall level of satisfaction with the course, assigning a grade from 1 to 5 , with 1 being "bad" and 5 being "excellent". As shown in Fig. 2(b), more than $80 \%$ of the students were roughly well satisfied, with over $55 \%$ of them expressing the maximum level of satisfaction with the course.

\section{CONCLUSIONS}

This paper described the work carried out during the last academic years to improve the quality of teaching and learning in an Electronic Materials course, which forms part of the Materials Engineering degree syllabus at the University of Seville. The results of this activity enabled students to broaden their knowledge of the contents related to Nanoelectronics through the use of the nanoHUB.org platform, the multimedia resources and remote computing tools of which were directly accessible via web links from the course's WebCT site. The combined use of both platforms, WebCT and nanoHUB.org, contributed to a more efficient exploitation of new teaching and learning methodologies.

\section{ACKNOWLEDGMENT}

The author would like to thank all the students on the Electronic Materials course, corresponding to the Materials Engineering degree of the University of Seville, for their help in those activities carried out to improve the quality of teaching during the academic year 2010-2011, and, in particular, Ms. Rocío Moriche-Tirado, whose collaboration in searching for and systematizing resources and in incorporating teaching materials into the WebCT platform was of invaluable assistance.

\section{REFERENCES}

[1] D. Pishva et al., "A Survey on How Blackboard is Assisting Educational Institutions Around the World and the Future Trends," Proc. of IEEE Int. Conference on Advanced Communication Technology (ICACT), pp. $1539-1543,2010$

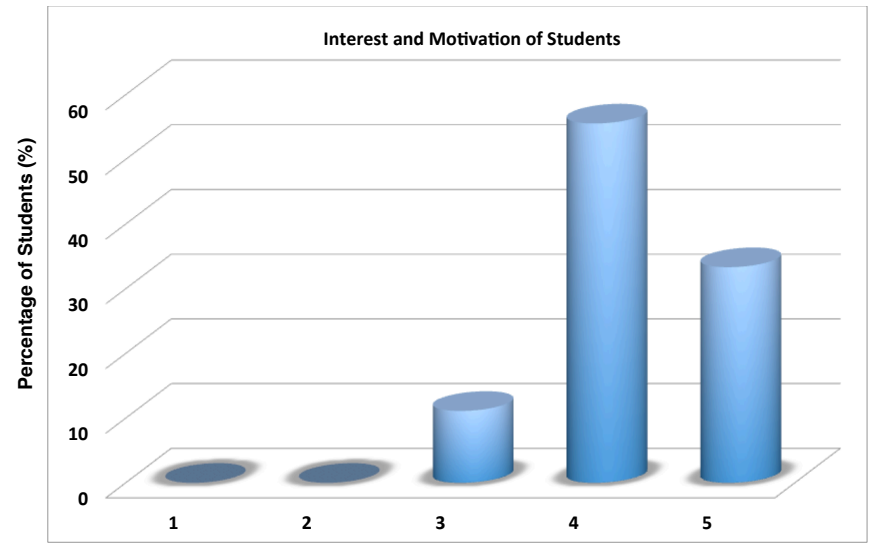

Motivation Level ( 1 = "Bad", 2 = "Average", 3= "Good", 4= "Very Good", 5="Excellent") (a)
[2] nanoHUB.org, On-line Simulation and More for Nanotechnology. [Online]. Available: http://nanoHUB.org.

[3] G. Klimeck et al., "nanoHUB.org - Online Simulation and More Materials for Semiconductors and Nanoelectronics in Education and Research," Proc. of IEEE Conf. on Nanotechnology, pp. 17-23, 2008.

[4] H. McNally, ECET 499N Lecture 10: Nanomaterials. [Online]. Available: http://nanohub.org/resources/8771.

[5] G. Klimeck et al., Quantum Dot Lab. [Online]. Available: http://nanohub.org/resources/450.

[6] A. Paul et al., Crystal Viewer Tool. [Online]. Available: http://nanoHUB.org/resources/3741.

[7] G. Seol et al., CNTbands. [Online]. Available: http://nanoHUB.org/resources/1838.

[8] Y. Zhao et al., CNT Mobility. [Online]. Available: http://nanoHUB.org/resources/6688.

[9] N. Neophytou et al., CNTFET Lab. [Online]. Available: http://nanohub.org/resources/1091.

[10] A. Rahman et al., FETToy. [Online]. Available: http://nanoHUB.org/resources/220.

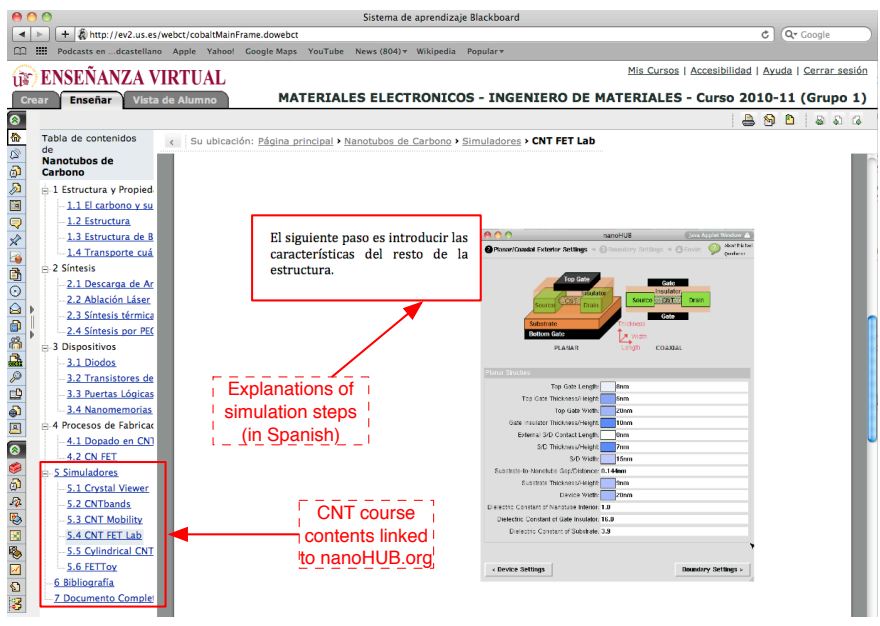

Figure 1. Illustrating some nanoHUB.org tools linked to the WebCT course.

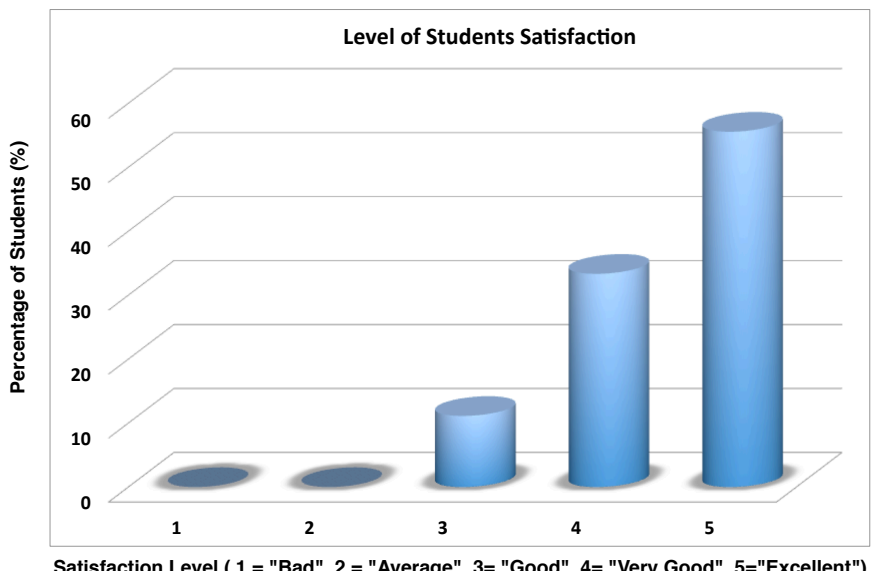

(b)

Figure 2. Students' feedback and results. (a) Interest and motivation for the course contents. (b) Overall satisfaction level. 